\title{
Comportamento organizacional e a sua relação com a implantação de um erp:
} uma revisão

\author{
Cristiano Geraldo Teixeira Silva ${ }^{1}$ \\ Mario Teixeira Reis Neto ${ }^{2}$
}

\section{Resumo}

A adoção de um ERP (Enterprise Resources Planning) na organização causa impactos positivos e negativos destacados por vários estudos. Os motivos para a implantação podem ser tecnológico, estratégico ou legal. O planejamento para a implantação é essencial para que a empresa obtenha benefícios com a efetivação do sistema de gestão na empresa. É preciso envolver a equipe de trabalho e ficar atento quanto aos anseios, expectativas e comportamentos neste processo. Muitas pessoas podem resistir à mudança por medo e, principalmente, por falta de comunicação sobre o futuro e as intenções da empresa. Diante disso, este estudo tem o objetivo de apresentar pesquisas sobre o comportamento organizacional frente à mudança causada pela implantação de um sistema ERP. São apresentados os conceitos e discutidas as relações entre eles, bem como as soluções apresentadas pelos autores para evitar riscos e explorar o máximo dos benefícios neste período de mudanças organizacionais.

Palavras-chave: Comportamento, Mudança Organizacional, ERP

\begin{abstract}
The ERP (Enterprise Resources Planning) adoption in the organization causes positive and negative impacts highlighted by several studies. The reasons for the deployment may be technological, strategic or legal. The planning for the implantation is essential to the company obtains benefits with the effectiveness of the management system in the company. It is necessary to involve the work team and to be attentive about the yearnings, expectations and behaviors in this process. Many people can resist change because of fear and especially because of lack of communication about the future and the intentions of the company. Therefore, this study aims to present research on organizational behavior in the face of the change caused by the implementation of an ERP system. It presents the concepts and discussed the relationships between them, as well as the solutions presented by the authors to avoid risks and to exploit the maximum benefits in this period of organizational changes.
\end{abstract}

Keywords: Behavior, Organizational Change, ERP

\section{Introdução}

Diante da atual exigência no mercado, as empresas adotam soluções para aumentar a sua produtividade com qualidade e baixo custo. Uma forma de alcançar este

\footnotetext{
${ }^{1}$ Doutor em Administração pela Universidade Federal de Minas Gerais. Professor do Programa de Mestrado e Doutorado em Administração da Universidade FUMEC. reisnetomario@ fumec.br

${ }^{2}$ Mestre em Tecnologia pelo Centro Federal de Educação Tecnológica de Minas Gerais. Professor da Universidade FUMEC. cgts@fumec.br
} 
objetivo é através do investimento em tecnologias, onde se destacam os softwares de gestão que são oferecidos às empresas sob uma forte argumentação para a melhoria dos processos administrativos (FURINI, 2014).

Segundo pesquisa realizada pela IDG Research Services, as empresas que utilizam um sistema de gestão empresarial têm crescimento 35\% mais rápido e produtividade $10 \%$ maior do que aquelas que não possuem acesso a soluções similares (KING et al., 2014).

Com o avanço das tecnologias para conexão via Internet, é possível perceber atualmente uma grande oferta de sistemas de gestão empresarial nas nuvens. Tais soluções proporcionam economia na implantação, pois exigem da empresa apenas máquinas conectadas à Internet. Esta solução traz benefícios na alimentação de dados e na obtenção on-line das informações para tomada de decisões. Além disso, oferece uma forte integração com o e-comércio, uma vez que este também depende da base de dados fornecida pelos sistemas integrados (PAVIN \& KLEIN, 2015).

A implantação de um ERP (Enterprise Resources Planning) favorece o desenvolvimento de melhores processos de trabalho, rastreabilidade, integração entre departamentos e maior agilidade na análise dos resultados. O uso de um ERP pode proporcionar à empresa resultados negativos, mas, principalmente, resultados positivos (DAVENPORT, 2002).

Apesar das vantagens destacadas com a adoção de um ERP, as organizações ainda enfrentam problemas na sua implantação. Algumas dessas dificuldades estão relacionadas aos aspectos comportamentais dos colaboradores, integrantes da equipe de implantação e executivos da empresa, envolvendo resistências a mudanças, comprometimento e dedicação da equipe de implantação, treinamento e motivação, comunicação dos objetivos e etapas do projeto, entre outras (BIEHL, 2007; KANSAL, 2006; SHEPHERD, 2006).

A partir das mudanças proporcionadas pela adoção de um ERP, a associação entre o comportamento individual e organizacional é um fator fundamental a ser considerado para obter sucesso através do comprometimento dos envolvidos. O comportamento organizacional estuda como as atitudes e o comportamento das pessoas nas empresas afetam o desempenho das organizações, bem como buscam soluções para aumentar a eficácia (SHMITT, 2004).

É possível identificar as seguintes razões que levam os usuários a adotarem o comportamento de resistência à implantação de um ERP: necessidade de revisão de 
processos, adoção de controles mais rígidos, uso de banco de dados único e centralizado, consumo de tempo que poderia ser dedicado a outros projetos ou atividades, maior customização do ERP comparativamente a sistemas legados, e perspectiva de redução do quadro funcional (CORTES \& LEMOS, 2009).

Diante da importância e escassa produção científica recente desta temática, principalmente na literatura de Administração, sua discussão torna-se relevante a luz do seu conceito nas reflexões sobre a relação entre implantação de ERP e comportamento organizacional, e apresenta-se como operativo, inovador e atual.

Assim, o presente artigo pretende discutir a relação entre o comportamento organizacional e a implantação de um ERP através de estudos anteriormente realizados e conceitos elaborados por diversos autores. Os conceitos e as discussões apresentadas favorecem o conhecimento na área e não tem a pretensão de esgotar o assunto, mas, contribuir para o surgimento de outros estudos.

\section{A Implantação de um ERP}

O software ERP é um sistema de informática responsável por cuidar de todas as operações administrativas e operacionais da empresa. Um benefício deste software é a integração das áreas de orçamento, execução, controladoria, finanças, gerenciamento de projetos, controle de estoque e recursos humanos. Para a sua implantação é necessário o conhecimento minucioso do fluxo de negócios e das atividades da empresa (PAVIN \& KLEIN, 2015).

A integração promovida pelo ERP corresponde a uma das perspectivas clássicas dos sistemas de informação que é a unificação e o tratamento centralizado de informações. Nesse contexto, cada componente do subsistema produz e absorve a informação, cuja preocupação com a eliminação de redundâncias e inconsistências é um dos passos da unificação dos dados. É o uso da informação que cria valor para a organização e não a tecnologia da informação isoladamente. À medida que o ERP se torna cada vez mais presente, faz-se necessário que as empresas se envolvam no processo de gestão da informação e, por meio dessa integração, realizem mudanças nos processos de negócio no setor produtivo da empresa (HASSABELNABY et al., 2012).

Os sistemas ERP encontram fundamentos na Teoria Geral dos Sistemas (TGS), desenvolvida pelo biólogo alemão Ludwig von Bertalanffy, segundo o qual um sistema pode ser definido como um complexo de elementos em interação (BERTALANFFY, 1975). A TGS ampliou a abrangência dos conceitos de sistema a partir dos enfoques 
social, institucional e político. Isso porque se torna mais fácil encarar os fenômenos quando analisados sob uma visão sistêmica. A relação da TGS com o sistema ERP é significativa, tendo em vista a necessidade de os sistemas ERP estarem presentes na organização como um todo e não somente em departamentos ou setores. Assim, os dados são coletados de forma sistemática, fundamentada pela teoria quando esta se refere a elementos que influenciam o processo como um todo (BATISTA et al., 2014).

Davenport (2002) também ressalta que os sistemas ERP são plataformas de software desenvolvidas para integrar os diversos departamentos de uma empresa, possibilitando a automação e o armazenamento das informações dos negócios. A arquitetura da Tecnologia da Informação (TI) de uma organização é um mapa ou plano de alto nível dos recursos de informação. Esta arquitetura precisa integrar os requisitos de informação da organização e de seus usuários para atender às necessidades estratégicas empresariais. O objetivo de um sistema ERP é suportar toda a informação administrativa exigida para a tomada de decisão dentro de uma organização. Adotar um sistema ERP possibilita a integração das áreas e setores funcionais da empresa a partir do compartilhamento dos dados (DAVENPORT, 2002).

Quando a implantação ocorre de forma planejada, é possível perceber melhoria na integração entre as áreas, o que é essencial para aprimorar o processo de comunicação da empresa e para promover melhor análise da empresa como um todo. A obtenção da informação de forma mais rápida e útil, a redução de retrabalho e maior acompanhamento de todos os trabalhos executados pelos setores são alguns resultados obtidos a partir da integração entre os departamentos (HASSABELNABY et al., 2012).

As últimas décadas têm sido marcadas por mudanças significativas em várias questões: expansão de mercados, rapidez de comunicações, disseminação de conhecimento e principalmente de novas e arrojadas tecnologias (FURINI, 2014). Nesse cenário, é necessário que as organizações tenham alta capacidade de adaptação com vistas à sua sobrevivência. Chiavenato (2014) aponta que o processo de mudança organizacional começa com o aparecimento de forças internas ou externas às empresas. As exógenas são provenientes do ambiente e as endógenas do comportamento organizacional. Uma das principais forças endógenas que uma organização pode enfrentar é a implantação de um novo sistema operacional informatizado. a partir das necessidades de adaptação ao cenário de mudanças, as organizações buscam o aprimoramento constante de suas gestões através da adoção de uma ferramenta 
considerada de desempenho satisfatório: os sistemas com a filosofia ERP (CHIAVENATO, 2014)

Mais que uma mudança tecnológica, o uso de um sistema de gestão ERP implica um processo de transformação organizacional. A implementação de um sistema integrado de gestão pressupõe que a organização realize diversas ações em sua estrutura, tanto operacionais quanto estratégicas, adequando internamente sua cultura organizacional, para a integração sinérgica entre a operação e a gestão da informação (FURINI, 2014).

Vasconcelos e Pinochet (2002) descrevem que a implantação de uma nova tecnologia, como por exemplo, um sistema integrado de gestão, implica em mudança de hábitos, comportamentos, estratégias e práticas sociais. Se dentro de uma empresa os indivíduos se organizam em grupos fechados, tais como "feudos", e não trocam informações entre si, a tendência é que a implantação do ERP não atinja os benefícios esperados. Portanto, novas regras organizacionais e sistemas de recompensa devem ser definidos para que os atores organizacionais mudem o seu comportamento e suas estratégias anteriores, e percebam que o novo sistema será "um bom negócio" tanto para eles quanto para a organização (PUENTE-PALACIOS et al., 2011).

Como a implantação de um ERP envolve o empenho dos funcionários da empresa e acompanha mudanças muitas vezes significativas, é relevante o conhecimento do comportamento organizacional e das soluções para promover uma maior eficácia neste processo.

\section{Valores e comportamentos na mudança}

O comportamento organizacional é uma área de estudos que investiga o impacto que indivíduos, grupos e a estrutura organizacional têm sobre o comportamento das pessoas dentro das organizações, com o objetivo de usar esse conhecimento para melhorar a eficácia organizacional. A mudança organizacional é um componente básico de estudo nesta área e ocorre em uma implantação de um ERP. As organizações bemsucedidas precisam fomentar a inovação e dominar a arte da mudança, mantendo a flexibilidade e continuidade no aprimoramento de sua qualidade. O desafio dos administradores é estimular os seus funcionários, promovendo o envolvimento nas atividades (ROBBINS et al. 2011). 
A promoção do grau de envolvimento dos colaboradores depende de uma comunicação eficaz, o tratamento de conflitos interdepartamentais e um planejamento de toda a condução (HUANG et al, 2004)

As dificuldades de implantação dos sistemas ERP decorrem, principalmente, pelo fato da implantação envolver mudanças organizacionais, alterações nas tarefas e nas responsabilidades de indivíduos e departamentos, além de transformações nas relações de poder entre os diversos departamentos da empresa. A mudança organizacional é um momento de ruptura, de transformação e criação, que geralmente envolve riscos, principalmente, o risco das alterações em relação ao poder. (COSTA, 2002; CALDAS \& WOOD JR, 1999).

O poder é apontado por vários estudos como um valor que influencia o comportamento do indivíduo (CALDAS \& WOOD JR, 1999; COSTA, 2002; SCHWARTZ, 1999). Segundo Schwartz (1999), o poder corresponde a status social e prestigio que se caracteriza no controle ou domínio sobre pessoas e recursos. Uma dimensão de dominação ou submissão surge na maioria das análises empíricas das relações interpessoais tanto dentro da organização quanto entre organizações. Para justificar esse fato da vida social e motivar os membros do grupo a aceitá-lo, os grupos devem tratar o poder como um valor. Os valores de potência também podem ser transformações das necessidades individuais de dominância e controle.

Gouveia (2003) separa o valor de prestígio e poder, mas, mantém uma relação direta entre eles. O prestígio é classificado conforme a importância do contexto social, não sendo uma questão de o indivíduo ser aceito pelos demais, mas de ter uma imagem pública. Os indivíduos que assumem este valor reconhecem a importância dos demais, desde que isso resulte em seu próprio benefício. Já o poder é menos social do que o prestígio e as pessoas que atribuem importância a ele podem não ter a noção de um poder socialmente constituído.

Além do prestígio estar relacionado ao status, é também válido o relacionamento com o respeito, valorizado pelo profissional. A diferença entre o status de organização (prestígio) e o status dentro da organização (respeito) é um aspecto entre os indivíduos, a ser compreendido para melhorar o processo de identificação organizacional, através de perspectivas multifaceadas, como o modelo de engajamento de grupo (FULLER et al., 2009).

O conhecimento sobre estudos relacionados aos valores individuais e grupais pode contribuir para o planejamento de mudanças como a implantação de um ERP. O 
estudo realizado por Schwartz (1999), que envolve a classificação de valores e a identificação destes em um grupo de pessoas, apresenta uma visão geral que reforça a importância dos valores na influência de comportamentos nas organizações. A teoria de valores especifica seis principais características: valores são crenças, valores se referem a metas desejadas, valores transcendem ações e situações, valores servem como padrões ou critérios, valores são ordenados pela importância, e a relativa importância de múltiplos valores orientando as ações. Segundo esta teoria, os valores fazem parte de três requerimentos: necessidades biológicas, requisitos para interação social e sobrevivência e bem-estar necessário a um grupo.

Conforme pode-se observar na figura 1 , os valores são agrupados em quatro partes compatíveis, de forma que a apresentação deste relacionamento também demonstra o conflito entre valores. A representação circular apresenta de forma clara a relação (perto) e o antagonismo (longe) entre os valores. A partir da implantação de um ERP, pode-se considerar que indivíduos com altos valores de conservação serão mais resistentes ao uso do software se comparados àqueles que tem maior valor de abertura à mudança (SCHWARTZ, 1999).

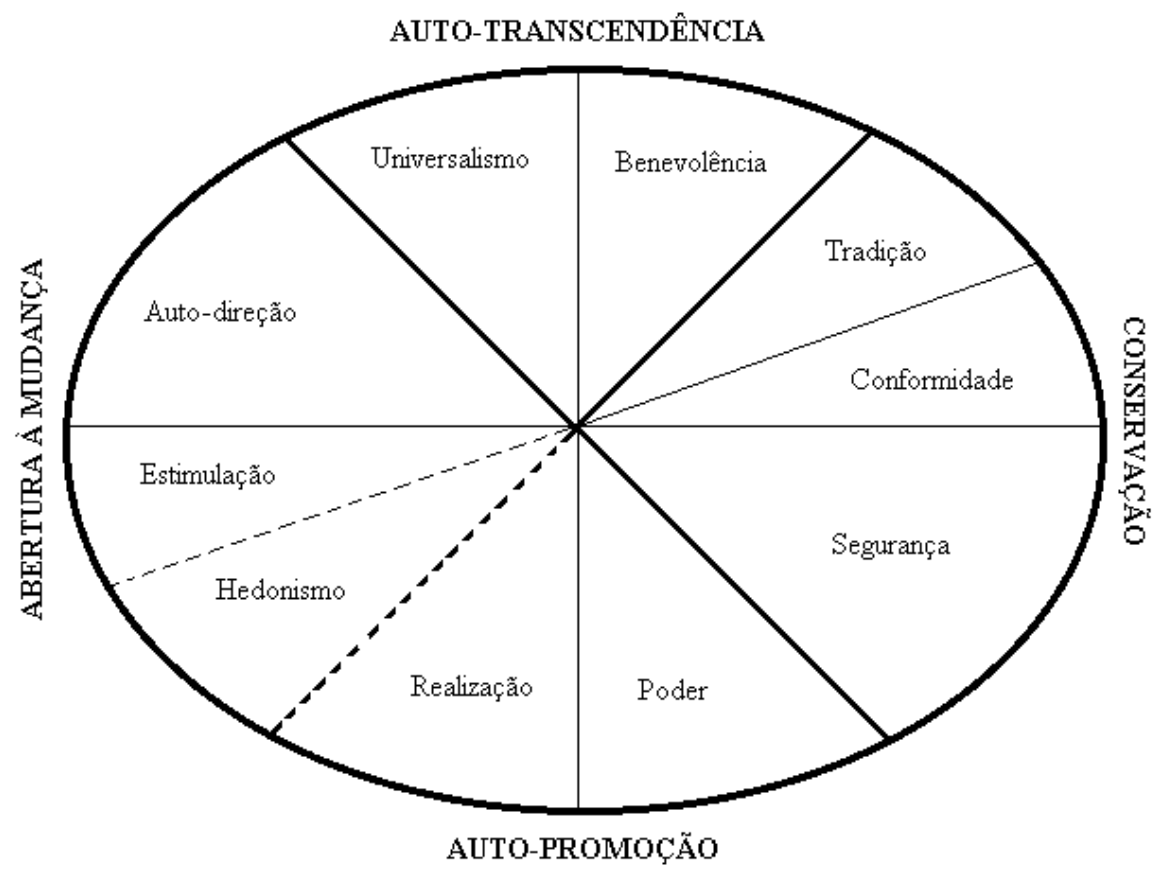

Figura 1: Valores conforme teoria de Schwartz (1999)

Fonte: SCHWARTZ (1999) 
Embora a teoria discrimine dez valores, é postulado que, em um nível mais básico, os valores formam um continuo de motivações relacionadas (SCHWARTZ, 1999). Para esclarecer a natureza do contínuo, observa-se a ênfase motivacional compartilhada de valores adjacentes:

- poder e realização: superioridade e estima social;

- realização e hedonismo: satisfação egocêntrica;

- hedonismo e estimulação: desejo de excitação afetivamente agradável;

- estimulação e auto direção: interesse intrínseco pela novidade e domínio;

- auto direção e universalismo: dependência do próprio juízo e conforto com a diversidade da existência;

- universalismo e benevolência: valorização dos outros e transcendência de interesses egoístas;

- benevolência e tradição: devoção ao seu próprio grupo;

- benevolência e conformidade: comportamento normativo que promove relações estreitas;

- conformidade e tradição: subordinação de si em favor de impostas socialmente expectativas;

- tradição e segurança: preservação das disposições sociais existentes que favorecem a vida;

- conformidade e segurança: proteção da ordem e harmonia nas relações;

- segurança e poder: evitando ou superando ameaças, controlando relacionamentos e recursos.

Diante das argumentações acima, é possível comparar a última relação apresentada entre segurança e poder como um motivo relevante às atitudes de resistência à mudança promovida pela implantação de um ERP.

\section{Resistência à mudança}

A resistência à mudança organizacional pode ser notada pelos comportamentos dos colaboradores que reagem à implantação de novas tecnologias, de novos sistemas de gestão e controle de informações organizacionais e de novas formas de comunicação hierárquica. A resistência é a reação comportamental que está associada ao medo do novo, à ameaça de perda do poder, à perda do cargo e à perda das vantagens de posicionamento nos fluxogramas organizacionais por onde passam informações 
privilegiadas. Um vínculo estrutural é um relacionamento dentro de uma rede social em que um participante se estabelece como um vínculo crítico entre indivíduos ou grupos inteiros. Isso permite que este indivíduo controle os fluxos de informações ou de recursos para a manutenção de vantagem própria. A tentativa de manutenção de um status, por alguns indivíduos, e seus comportamentos de resistência à mudança impedem o sucesso da organização em adquirir e operar por meio de novas tecnologias (VASCONCELOS \& PINOCHET, 2002; COSTA, 2002; BHATTI, 2005).

Ayres (2000) ressalta que estas mudanças se manifestam de duas maneiras distintas: (a) a resistência para aceitar a tecnologia e (b) a forma de se identificar com a tecnologia. $\mathrm{O}$ autor menciona que a resistência para aceitar a tecnologia pode resultar em comportamentos passivos ou, em casos mais graves, em sabotagens, enganos intencionais, recusa para usar o sistema e tentativas de torná-lo inoperante. O esforço despendido e a ansiedade sentida pelos indivíduos, que têm que se adaptar aos processos de mudanças organizacionais, gera níveis gradativos de stress e chegam a causar mudanças de comportamento nestes indivíduos (JARAMILLO et al., 2011).

O stress psicológico causa nos indivíduos medo, pânico, ansiedade, frustração, preguiça mental, falta de concentração, impaciência e depressão. A rapidez com que as mudanças acontecem, sobretudo as que envolvem sistemas de informação, e a forma como elas são comunicadas dentro da estrutura, são fatores que podem desencadear estados gradativos de stress psicológico. O stress, um fenômeno inevitável, torna-se altamente negativo quando os indivíduos têm dificuldades para se adaptarem ao novo ambiente (AYRES, 2000; OLIVEIRA et al., 2013).

O stress está relacionado positivamente com o conflito interpessoal. A concorrência interna e as divergências de interesses afetam os relacionamentos com os colegas de trabalho. O conflito interpessoal influenciará em exaustão emocional e, consequentemente, causará o stress no indivíduo (JARAMILLO et al., 2011).

A dedicação integral da equipe responsável pela implantação do ERP é um fator relevante. Em geral, parte dessa equipe acumula outras funções dentro da organização, gerando stress nos envolvidos e acarretando no risco de rotatividade entre os integrantes. Este é um fator comportamental que precisa ser adequadamente tratado, pois está relacionado ao comprometimento do desempenho da equipe e aos impactos negativos que podem ser causados na implantação do ERP (CORTES \& LEMOS, 2009). 
A sobrecarga de trabalho também está relacionada positivamente com o stress. $\mathrm{O}$ indivíduo sobrecarregado tende a se envolver em conflitos interpessoais e, consequentemente terá maior susceptibilidade ao stress (JARAMILLO et al., 2011).

\section{Práticas para o comprometimento}

Um fator que influencia no comportamento organizacional para a efetiva implantação do ERP corresponde à conscientização e ao comprometimento da alta administração da empresa. Sem esse comprometimento o projeto não deve ser executado, pois é de fundamental importância que os gestores ofereçam as envolvidos uma atmosfera de confiabilidade e uma clareza de foco. É preciso obter o envolvimento de todos os profissionais, informando-os do andamento das tarefas, cobrando atualização de cronograma e utilizando táticas de esclarecimentos dos objetivos de cada atividade (CORTES \& LEMOS, 2009).

A afirmação acima está relacionada à necessidade de realizar um trabalho inteligente através de um comportamento adaptável às mudanças e de realizar um planejamento das atitudes conforme as adversidades para evitar o risco de stress no trabalho (JARAMILLO et al., 2011).

As práticas para o gerenciamento da equipe envolvem a utilização de comunicações mais direcionadas e algumas práticas de recursos humanos para promover alto comprometimento com a empresa. O gestor deve conhecer sua equipe para aplicar estas técnicas e conciliar as expectativas dos colaboradores (FULLER et al., 2009).

Os treinamentos e a troca de experiências vividas em implantações podem contribuir para o processo de mudança na organização. Estudos destacam o treinamento como uma solução para mudar crenças e estimular a aceitação dos objetivos da empresa (BORG, 2001; FULLER et al., 2009). Como as crenças podem motivar o indivíduo nas suas ações, um treinamento pode tornar o funcionário mais disposto a exercer um esforço considerável pelos benefícios do ERP e a se manter como membro da instituição.

Cada indivíduo pode vir a expressar diferentes níveis de comprometimento. Isso se deve às características de seu comportamento, da satisfação com o que realiza, de seu bem-estar, dos benefícios oferecidos, da internalização dos valores e dos objetivos organizacionais propostos. Também está relacionado ao envolvimento afetivo e das oportunidades que a empresa possa vir a oferecer (VARGAS, 2012). 
Outro importante fator para promover um maior comprometimento é a liderança transformacional. As atitudes dos líderes a partir do carisma e influência, motivação, estimulação intelectual e consideração individualizada são importantes para promover o comprometimento nas organizações. O carisma e a influência correspondem aos líderes que possuem uma meta idealizada, têm compromisso pessoal para alcançá-la e são modelos para os seus seguidores. A motivação inspiracional descreve líderes que comunicam altas expectativas para seus seguidores, inspirando-os, motivando-os a serem comprometidos com a visão compartilhada da organização e realçam o espírito de equipe. A estimulação intelectual corresponde a líderes que estimulam os seus seguidores a serem criativos e inovadores, fazendo com que desafiem as próprias opiniões, as de seu líder e as de sua organização, com o propósito de pensar novas maneiras e desenvolverem novos caminhos para os negócios. Por fim, a consideração individualizada corresponde aos líderes que escutam seus seguidores, que fornecem suporte, que buscam ajudar, aconselhar e que tratam cada empregado de maneira única. Este líder pode dar uma forte afiliação e também dar direções específicas para seus seguidores (MARQUES, 2007).

Conforme abordagem de vários estudos, é possível constatar que o conhecimento do comportamento organizacional é importante para a implantação de sistemas ERP, uma vez que corresponde a um processo de mudança organizacional (CARVALHO \& JOHANSSON, 2009; LAW \& NGAI, 2007; PAVIN \& KLEIN, 2015; WIER et al., 2007). Além disso, aplicar práticas que promovem o envolvimento e o comprometimento da equipe é uma solução eficaz para o sucesso da implantação.

\section{Considerações finais}

A implantação dos sistemas ERP nas organizações exige uma análise cuidadosa de fatores estratégicos que garantam o sucesso do projeto e o alcance dos objetivos organizacionais para os quais a decisão de implantar o sistema foi tomada. A integração dos departamentos da empresa é uma tarefa que envolve o detalhamento dos processos organizacionais e dos objetivos da empresa. Portanto, os procedimentos internos devem estar bem elaborados e também abertos a qualquer mudança, pois a tecnologia é base de sustentação da organização.

Além do planejamento e do controle sobre os impactos tecnológicos e procedimentais que um novo sistema de gestão pode proporcionar à empresa, é necessária a atenção quanto ao envolvimento da equipe de trabalho. Conforme abordado 
neste artigo, estudos comprovam que o planejamento, a conscientização e a comunicação são fatores essenciais para o efetivo sucesso da implantação de um ERP. Manter a equipe motivada durante um processo de mudança requer acompanhamento constante e medições que vão deste o andamento físico do projeto quanto às expectativas dos seus colaboradores.

Este estudo aborda as questões emocionais e comportamentais dos indivíduos em situações de mudança organizacional, mais especificamente, na implantação de um ERP. As abordagens selecionadas visaram uma revisão sobre este assunto para apoiar o conhecimento e estimular o desenvolvimento de outras pesquisas. Outros estudos podem ser realizados com o propósito de obter modelos comportamentais conforme variedade de metodologias de implantação. Um exemplo é a oferta atual no mercado de ERP via Internet mais acessível às pequenas e médias empresa. Algumas destas soluções se baseiam na condução, quase que única, do gestor da empresa, sob a característica de fácil utilização da ferramenta. Seria relevante estudos para avaliar se este tipo de solução garante sucesso na implantação. Outra pesquisa importante seria trabalhar a disponibilidade dos gestores e o comportamento organizacional favorável à condução de uma implantação de um software apenas com um treinamento à distância ou, simplesmente com uso de uma ferramenta oferecida no mercado com o benefício em facilidade de utilização. Além disso, outros estudos para avaliar o uso de práticas motivacionais envolvendo pequenas e médias empresas na implantação de ERP seriam importantes ao ambiente profissional e acadêmico.

\section{Referências}

AYRES, K. V. Tecno-stress: um estudo em operadores de caixa de supermercado. In: $24^{\circ}$ Encontro Nacional de Programas de Pós-Graduação - ENANPAD, Florianópolis, 2000.

BATISTA, G.; MURBACK, F. G. R.; SARES, M. I. F.. Estudo sobre a Aplicação da Teoria Geral dos Sistemas nas Organizações Brasileiras. Décimo Congresso Brasileiro de Sistemas. Ribeirão Preto, 2014. 30p.

BERTALANFFY, V. L.; BILLAH, A. B. M. M.; PUTTEE,C.; ALI, Y. Consideration of Critical Success Factors for Enterprise Resource Planning Systems Implementation in Context of Bangladesh. Enterprise Resource Planning. Petrópolis: Editora Vozes, 1975.

BHATTI, T. R. Critical success factors for the implementation of enterprise resource planning (ERP): empirical validation. Second International Conference on Innovations in Information Technology. Dubai, UAE, set. 2005. 10 p. 
BORG, M.. Teachers' Beliefs. ETL Journal. v. 55, nº 2, pp. 186-188, Abril, 2001.

CALDAS, M. P.; WOOD JÚNIOR, T. Modas e modismos em gestão: pesquisa exploratória sobre adoção e implantação de ERP. Foz do Iguaçu. Anais do Enanpad 1999, 1999.

CARVALHO, R. A.; JOHANSSON, B. Enterprise resource planning systems for small and medium enterprises. Handbook of research on software engineering and productivity technologies: Implications of globalisation. IGI Global. pp. 373-381, 2009.

CHIAVENATO, Idalberto. Introdução à teoria geral da administração. 9. ed. São Paulo: Manole, 2014, 650 p.

CORTES, P. L.; LEMOS, M. T. S.. Aspectos comportamentais na implantação de sistemas integrados de gestão - ERP. GEPROS - Gestão da Produção, Operações e Sistemas, v. 4, nº 1, pp. 83-100. Jan-Mar, 2009.

COSTA, L. S. A. B. Benefícios percebidos com a implantação do ERP III / SAP: um estudo comparativo de casos. In: Encontro Nacional de Pós-graduação em Administração, n. 26, Salvador. Anais Rio de Janeiro: ANPAD, 2002.

DAVENPORT, T. H. Missão crítica: obtendo vantagem competitiva com os sistemas de gestão empresarial. Porto Alegre: Bookman, 2002.

FULLER, J. B.; HESTER, K.; BARNETT, T.; BEU, D.; FREY, L.; RELYEA, C.. Extending the Group Engagement Model: An Examination of the Interactive Effects of Prestige, Respect, and Employee Role Identity. Journal of Managerial Issues. v.21, $\mathrm{n}^{\circ}$ 1, pp.119-139. 2009.

FURINI, L. R. Benefícios obtidos após implantação de sistemas ERP. 2014. 92 p. Dissertação (Mestrado em Administração) - Universidade do Grande Rio, 2014.

GOUVEIA, V. V.. A natureza motivacional dos valores humanos: evidências acerca de uma nova tipologia. Estudos de Psicologia, v. 8, nº 3, pp. 431-443, 2003.

HASSABELNABY, H. R.; HWANG, W.; VONDEREMBSE, M. A.. The impact of ERP implementation on organizational capabilities and firm performance. Benchmarking: An International Journal, v. 19, n. 4/5, p. 618-633, 2012.

HUANG Shi-Ming, CHANG I-Shu, LI Shing-Han, LIN Ming-Tong. Assessing Risks in ERP Projects: identify and prioritize the factors. Industrial Management \& Data Systems. v. 104, no 8, pp. 681-688. Agosto, 2004.

JARAMILLO, F.; MULKI, J. P.; BOLES J. S.. Workplace Stressors, Job Attitude, and Job Behaviors: Is Interpersonal Conflict the Missing Link? Journal of Personal Selling \& Sales Management, v.31, nº 3, pp. 339-356. 2011. 
KING, J.; MCKEAN, J.; LABERIS, P.. Impact of data effectiveness on business outcomes at medium-sized businesses in North America and Europe. IDG Research Services, 2014.

LAW, C.C.; NGAI, E.W. . An investigation of the relationships between organizational factors, business process improvement, and ERP success. Benchmarking: An International Journal, v. 14, no 3, pp. 387-406. 2007.

MARQUES, G. M.; MEDEIROS, C. A. F.; FRANÇA, A. G. C.; RIBEIRO, M. F. G. R.. Estilos de liderança e comprometimento organizacional: uma aplicação Multifactor leadership Questionnaire no Brasil. Revista ADM.MADE, v.11, $n^{o}$ 2, pp.99-125. Mai/Ago 2007.

OliveirA, L. B.; CAVAZOTTE, F. S. C. N.; PACIELlO, R. R.. Antecedentes e Consequências dos Conflitos entre Trabalho e Família. RAC: Rio de Janeiro, v. 17, $\mathrm{n}^{\circ} 4$, pp. 418-437, Jul/Ago 2013.

PAVIN, R. D. P.; KLEIN, A. Z.. Organization consequences of the adoption of mobile ERP systems: Case studies in Brazil. JISTEM: São Paulo, v. 12, n' 2, p. 219-232, May/Aug 2015.

PUENTE-PALACIOS, K.; ALMEIDA, R. S.; REZENDE, D. V.. O Impacto da Interdependência no Trabalho sobre a Efetividade de Equipes. $O \& S$ : Salvador, v.18, ${ }^{\circ}$ 59, p. 605-623, Out/Dez 2011.

ROBBINS, S. P.; JUDGE, T. A.; SOBRAL, F.. Comportamento Organizacional. $14^{\mathrm{a}}$ ed. São Paulo: Pearson Prentice Hall, 2011.

SCHWARTZ, S. H.. A Theory of Cultural Values and Some Implications for Work Applied Psychology: an International Review. v. 48, nº 1, pp. 23-47. 1999.

SHMITT, C. A. Sistemas Integrados de Gestão Empresarial: 2004. 283 p. Tese (Doutorado em Engenharia de Produção) -Universidade Federal de Santa Catarina. 2004.

VARGAS, K. S.; BIRRER, J. A.; MINELLO, I. F.. Estilos de Aprendizagem e Níveis de Comprometimento Organizacional: Uma Abordagem com Residentes Multiprofissionais da Saúde. Rev. Adm. UFSM, v. 5, no 3, pp. 589-606, Set/Dez 2012.

VASCONCELOS, I. F. F. G., PINOCHET, L. H. C. Poder, Tecnologia e Controle Burocrático: Uma Análise Crozeriana em uma Empresa de Informática Paranaense. In: II ENEO - Observatório da Realidade Organizacional, 2002, Recife. Anais do II ENEO, 2002.

WIER, B.; HUNTON, J.; HASSABELNBY, H.R.. Enterprise resource planning systems and non-financial performance incentives: The joint impact on corporate performance. International Journal of Accounting Information Systems, v. 8, $\mathrm{n}^{\mathrm{o}} 3$, pp. 165-190. 2007. 